\section{$S_{\text {pco: }}$ : Let's Not Throw the Baby Out With the Bath Water}

\section{To the editor:}

I read the interesting study by Weaver et $\mathrm{al}^{1}$ and the accompanying editorial by Wilcox and Richards ${ }^{2}$ on noninvasive carbon monoxide (CO) detection in the February 2013 issue of RESPIRATORY CARE. I agree wholeheartedly with those authors that $\mathrm{CO}$ poisoning is an important public health concern, confounded by nonspecific symptoms that challenge diagnosticians. My experience with noninvasive measurement of carboxyhemoglobin by pulse oximetry $\left(\mathrm{S}_{\mathrm{pCO}}\right)$ with the RAD-57 (Masimo, Irvine, California), since FDA approval in 2006, both in hospital settings and in fire and emergency medical services, has been accompanied by continual improvements in both the sensitivity and specificity of the technology. As a scientist and author of protocols synthesized from available clinical evidence to guide use of $\mathrm{S}_{\mathrm{pCO}}$ by prehospital providers and firefighters, ${ }^{3}$ I would like to offer some observations on the study by Weaver and colleagues.

The purpose of the study was to provide information about the false positive rate of the RAD-57 pulse CO-oximeter. ${ }^{1}$ The manufacturer's accuracy specification is $\pm 3 \%$ at one standard deviation, meaning that $68 \%$ of measurements will fall within one standard deviation ( $\pm 3 \%$ ), while, according to the empirical rule for normally distributed data, $33 \%$ would fall outside one standard deviation in either direction ( \pm up to $6 \%$ ). Indeed, Weaver et al found that the RAD-57 functioned within the manufacturer's published specifications. Yet the restrictive definition of a false positive chosen by Weaver and colleagues $\left(\mathrm{S}_{\mathrm{pCO}}\right.$ at least $3 \%$ greater than carboxyhemoglobin $[\mathrm{COHb}]$ or $\mathrm{S}_{\mathrm{pCO}}>6 \%$ with a $\mathrm{COHb} \leq 6 \%$ in a non-smoker) did not reflect the same accuracy specification. In fact, this definition was tighter than that in an early study by Touger et $\mathrm{al},{ }^{4}$ who reported a $48 \%$ device sensitivity in 120 patients, and a recent study of 1,578 patients by Roth et al, ${ }^{5}$ that yielded a $94 \%$ sensitivity.

As Weaver suggests, the reference COoximeters also have an accuracy specification that most certainly may have influenced the comparisons between the RAD-57's $\mathrm{S}_{\mathrm{pCO}}$ readings and the laboratory $\mathrm{COHb}$ values. Not cited, but a potential explanation for the findings of Weaver et al is the work of Mahoney and colleagues, who com- pared CO-oximetry to gas chromatography, and found that the CO-oximeters typically under-reported $\mathrm{COHb}$ at values above $2.5 \%$, and over-reported $\mathrm{COHb}$ at values below $2.5 \% .^{6}$

Lastly, Weaver et al reported on 3 occult $\mathrm{CO}$ poisoning cases in non-smokers with measured $\mathrm{S}_{\mathrm{pCO}}$ values of $0 \%$ but $\mathrm{COHb}$ values of $>10 \%$. While those are not false positives, that discrepancy is certainly disconcerting and suggestive of false negatives, despite the correct assertion by Weaver et al that their study lacked the power to determine a false negative rate. I believe this highlights a key issue, discussed by Weaver et al, involving technician technique. The RAD-57 operator's manual ${ }^{7}$ highlights several important characteristics of $\mathrm{S}_{\mathrm{pCO}}$ technology that can cause erroneous readings: selection of correct sensor size, sensor placement, and ambient light. As described in the operator's manual, I have observed that misplacement of the sensor, or use of a regular sensor on a very slender digit, appears to allow some of the multiple wavelengths of light to completely bypass the digit, resulting in a zero $\mathrm{S}_{\mathrm{pCO}}$ reading, while others pass through the digit, yielding accurate heart rate and oxygen saturation values. While I have found that firefighters and emergency medical services providers who are frequent users of $\mathrm{S}_{\mathrm{pCO}}$ are well acquainted with both sensor placement and ambient light issues, it has not been my experience that our colleagues in the clinical community share that understanding of the operating characteristics of $\mathrm{S}_{\mathrm{pCO}}$. Since the introduction of the RAD-57 in 2006 there have been multiple improvements in the sensor design. It is my understanding that Masimo has included finger misalignment recognition in the most recent sensor version.

I fondly remember the first portable pulse oximeters that appeared in hospital wards and ambulances in the early 1980s. These were very temperamental devices, subject to interference from motion, low perfusion, ambient light, electrical current fluctuations, and calibration drift. Their sensitivity and specificity were initially terrible, ${ }^{8}$ but reached $\pm 3 \%$ by the 1990 s. There were no calls suggesting that pulse oximetry had insufficient evidence for broad clinical use, as Wilcox and Richards suggested about $\mathrm{S}_{\mathrm{pCO}}{ }^{2}$ Rather, clinicians embraced pulse oximetry, with its inherent limitations, as a valuable tool. Certainly, no modern-day clinician would base their assessment of pulse oxi- metry on its first-generation technology. Likewise, I would encourage readers to recognize that $\mathrm{S}_{\mathrm{pCO}}$ was born in 2006 and offers a valuable and continually improving screening tool for $\mathrm{CO}$ poisoning. ${ }^{9}$ The variability of the results reported in the literature reflects the evolution of the technology and in clinician understanding of how to use it. No screening device should be used in isolation when making patient care decisions. Clinical assessment and confirmation of suspicions with appropriate laboratory testing is imperative, as Weaver et al aptly point out.

Despite years of serum potassium measurement in laboratories around the world, blood collection (phlebotomy) and laboratory error remain the leading causes of erroneously reported hyperkalemia. ${ }^{10}$ There can be no substitute for clinician judgment and insight into the proper operation of medical equipment. To believe that any instrument used by humans will completely correct for operator error is wishful thinking, at best. The RAD-57, like any medical device, can suffer from operator error. Rather than condemning a new technology, I would suggest we strive to educate users on its proper operation and limitations, work with manufacturers to improve accuracy, and be certain not to overly rely on technology in lieu of clinical assessment and judgment.

Mike McEvoy PhD RN NRP Division of Cardiothoracic Surgery Albany Medical Center Albany, New York and Saratoga County Office of Emergency Services Ballston Spa, New York

Mr McEvoy has disclosed relationships with Masimo and Physio-Control.

\section{REFERENCES}

1. Weaver LK, Churchill SK, Deru K, Cooney D. False positive rate of carbon monoxide saturation by pulse oximetry of emergency department patients. Respir Care 2013; 58(2):232-240.

2. Wilcox SR, Richards JB. Noninvasive carbon monoxide detection: insufficient evidence for broad clinical use. Respir Care 2013;58(2):376-379.

3. McEvoy M. Sneak attack: what makes carbon monoxide so insidious? JEMS 2010; 10(Suppl):4-9. 
4. Touger M, Birnbaum A, Wang J, Chou K, Pearson D, Bijur P. Performance of the RAD-57 pulse co-oximeter compared with standard laboratory carboxyhemoglobin measurement. Ann Emerg Med 2010;56(4): 382-388.

5. Roth D, Herkner H Schreiber W, Hubmann N, Gamper G, Laggner AN, Havel C. Accuracy of noninvasive multiwave pulse oximetry compared with carboxyhemoglobin from blood gas analysis in unselected emergency department patients. Ann Emerg Med 2011;58(1):74-79.

6. Mahoney JJ, Vreman HJ, Stevenson DK, Van Kessel AL. Measurement of carboxyhemoglobin and total hemoglobin by five specialized spectrophotometers (COoximeters) in comparison with reference methods. Clin Chem 1993;39(8):16931700.

7. Masimo Corporation. RAD-57 operator's manual. Masimo Rainbow SET signal extraction pulse CO-oximeter. 2006.

8. Severinghaus JW, Kelleher JF. Recent developments in pulse oximetry. Anesthesiology 1992;76(6):1018-1038.

9. Bledsoe B, McEvoy M. Where there's CO; there's not always fire: how pulse COoximetry serves as an important assessment and triage tool. JEMS 2009;34:5-8.

10. Stankovic AK, Smith S. Elevated serum potassium values: the role of preanalytic values. Am J Clin Pathol 2004;121(Suppl 1):S105-S112.

DOI: $10.4187 /$ respcare. 02737

\section{$\mathrm{S}_{\mathrm{pco}}$ : Let's Not Throw the Baby Out With the Bath Water-Reply}

In reply:

We thank Dr McEvoy for his thoughtful reply to our report. He is correct that our definition of false positive was restrictive; however, we based this definition on the manufacturer's stated accuracy specification, and, indeed, found that the RAD-57 functioned as specified. When broadened to include 2 standard deviations $(95 \%$ of the data), the accuracy range would be $\pm 6 \%$, a range that is challenging for the purpose of diagnosing $\mathrm{CO}$ poisoning.

We readily acknowledge that technician technique may play a role in obtaining accurate data, even though our technicians and study team were trained by the manufacturer on probe placement. Our concern is that, whether by technical limitations or operator error, the RAD-57 may provide an erroneously low $\mathrm{S}_{\mathrm{pCO}}$ measurement in a patient with $\mathrm{CO}$ poisoning. We agree that $\mathrm{S}_{\mathrm{pCO}}$ technology can be valuable in broadly screening for occult $\mathrm{CO}$ poisoning. We offer that an elevated $\mathrm{S}_{\mathrm{pCO}}$ should raise concern about $\mathrm{CO}$ poisoning, especially if the evaluating clinician has not considered $\mathrm{CO}$ exposure. However, we strongly caution against using $\mathrm{S}_{\mathrm{pCO}}$ measurement to rule out $\mathrm{CO}$ poisoning when symptoms and circumstances suggest it. Returning a misdiagnosed patient to the scene of the poisoning can have devastating and even deadly consequences.

\section{Lindell K Weaver MD Susan K Churchill APRN-NP Kayla Deru \\ Hyperbaric Medicine LDS Hospital \\ Salt Lake City, Utah}

The authors have disclosed relationships with SciMetrika and Masimo.

DOI: $10.4187 /$ respcare. 02760

\section{$S_{\text {pco: }}$ : Let's Not Throw the Baby Out With the Bath Water-Reply}

\section{In reply:}

The study by Weaver and colleagues ${ }^{1}$ demonstrated that, while the RAD-57 COoximeter operated within the manufacturer's specifications, with $68 \%$ of $\mathrm{S}_{\mathrm{pCO}}$ measurements falling within $\pm 3 \%$ of the laboratory carboxyhemoglobin $(\mathrm{COHb})$ measurements of $<40 \%$, in several cases the $\mathrm{S}_{\mathrm{pCO}}$ reported by the RAD-57 underestimated the $\mathrm{COHb}$. This raises concerns about the utility of the RAD-57 in identifying cases of occult $\mathrm{CO}$ poisoning, which is one of the primary potential benefits of a point-of-care noninvasive carboxyhemoglobin screening test. Furthermore, Weaver et al's findings were consistent with other studies of the RAD-57.2-4

In response to Weaver et al's study, Dr McEvoy opines that these failures of the RAD-57 to report a $S_{\mathrm{pCO}}$ consistent with the laboratory $\mathrm{COHb}$ measurement could have been due to technician technique. The concern has previously been raised by another industry representative, ${ }^{5}$ in response to a prospective study that demonstrated wide limits of agreement and poor sensitivity of the RAD-57.2 While technique may have been a contributor to the discrepancy between $\mathrm{S}_{\mathrm{pCO}}$ and $\mathrm{COHb}$ in Weaver et al's study, this does not excuse the failure of the RAD-57 to identify elevated $\mathrm{COHb}$ levels. The use of any medical device is not isolated from user technique or user error, and dismissing false negative results described by Weaver et al and others as being due to poor technique ignores the potential consequences of broadening the clinical use of the RAD-57. If false negative values were obtained under relatively idealized settings (technicians were trained by industry representatives and were obtaining measurements in the setting of a research study), it is reasonable to assume that the rate of false negatives will not be lower in the nonidealized setting of real world clinical medicine, where attention to technique may be less meticulous than in a research study.

Given these considerations, the poor sensitivity, rate of false negatives, and the inaccuracy of the RAD-57 should be a warning to medical personnel that $\mathrm{S}_{\mathrm{pCO}}$ is not definitive, and that a normal $\mathrm{S}_{\mathrm{pCO}}$ should not be reassuring. Ultimately, the false negatives obtained by the RAD-57, whether due to technician technique, intrinsic device inaccuracies, or patient-level factors, demonstrates that the RAD-57 is not suitable as a screening device and that there is potential for measurement inaccuracies and patient harm in real world clinical settings.

We agree that further work to develop an accurate, precise, user-friendly, and noninvasive $\mathrm{S}_{\mathrm{pCO}}$ monitor is warranted, and that a rapid, accurate, point-of-care carbon monoxide monitor would be extremely valuable. The RAD-57 monitor, however, does not meet these criteria, based on the available clinical data, and there is insufficient evidence for its broad clinical use. ${ }^{1-4}$

Jeremy B Richards MD MA Pulmonary, Critical Care, and Sleep Medicine

Beth Israel Deaconess Medical Center Boston, Massachusetts

Susan R Wilcox MD

Department of Anesthesia and Critical Care

Massachusetts General Hospital Boston, Massachusetts

The authors have disclosed no conflicts of interest.

\section{REFERENCES}

1. Weaver LK, Churchill SK, Deru K, Cooney D. False positive rate of carbon monoxide 